\title{
Modifications to Membrane Heater Holders to Enable XEDS in the AEM
}

\author{
N. J. Zaluzec ${ }^{1}$, A. Janssen ${ }^{2}$, M. G. Burke², D. Gardiner ${ }^{3}$, S. Walden ${ }^{3}$ \\ 1. Electron Microscopy Center, NST Div, Argonne National Laboratory, Argonne, Il, USA \\ 2. Materials Performance Centre, University of Manchester, Manchester M13 9PL, UK \\ 3. Protochips Inc, Raleigh NC. USA
}

Membrane and furnace technology is frequently used today for in situ holder construction to enable elevated temperature TEM/STEM studies. Today, it is becoming increasingly important to not only visualize temperature dependent microstructural evolution but also quantify elemental changes. While both technologies provide high and stable temperatures, neither in their standard configurations readily facilitate X-ray Energy Dispersive Spectroscopy (XEDS) principally due to their sidewall penumbras [1], which can partially or completely block x-ray signal detection similar to that in liquid cell holders [2]. This penumbra can be most easily appreciated by reference to Figure 1. In this work we modified a membrane holder as illustrated in Figure1c and tested it in an FEI Tecnai F20, Tecnai F30 and CM200F TEMs equipped with windowless SDD systems from either EDAX (Apollo) or Oxford (Xmax).

To measure the relative performance of a given design, we use a $100 \mathrm{~nm}$ thick $\mathrm{SiN}_{\mathrm{x}}$ window on 200-300 $\mu \mathrm{m}$ Si frame, which is inserted and centered in the holder. The penumbra effect can be assessed by measuring the dependence of the SiK line intensity as a function of holder tilt. In Figure 2a shows the results from a unmodified membrane holder while $2 b$ in a modified configuration. In $2 a$, one can see the lack of counts at lowest tilts and the clear break in the slope of the Intensity vs Tilt curve where the penumbra no longer limits the detector solid angle at $\sim 25^{\circ}$. The initial nearly linear variation is due to the shadowing of the detector by the penumbra. Once the full detector solid angle is no longer limited then the variation for a uniformly thick film will vary as the thickness along the incident beam direction. For this geometry the intensity variation will be an inverse cosine function of tilt $\left(t^{*}=t / \cos (\theta)\right)$. This can be seen in Figure $2 b$ and is not detectable in $2 a$, due to the extremely limited operational regime. Due to their dimensions and limited space in TEM pole piece gaps, there is little that can be done with furnace heaters until their size is reduced, and results from testing that type of holder are not shown here.

Whereas adapting a membrane holder to minimize the penumbra has been successful the application of windowless XEDS to high temperature studies also requires one to establish the limitations due to infrared (IR) radiation emitted by the thermal heating. Figure 3 shows that up to $400^{\circ} \mathrm{C}$ there is no detectable difference; however, above $450^{\circ} \mathrm{C}$ a gradual distortion (Figure 4) due to detection of the IR signal because of the absence of an absorbing window is clear. Above $525^{\circ} \mathrm{C}$ unless the detector is protected from IR by a suitable window, the spectroscopy for a windowless SDD is not realizable in our systems. Further work is currently in progress to investigate low background (Be) shields as a means to mitigate system peaks arising from the holder body.

References:

[1] Zaluzec N.J. Microsc. Microanal. 20 (2014), p. 1318.

[2] Zaluzec N.J et al, Microsc. Microanal. 20(2) (2014), p. 323.

[3] Research supported by the U.S. DoE, Office of Science, Office of Basic Energy Sciences, under Contract No. DE-AC02-06CH1 1357 at the Electron Microscopy Center, Nanoscience and Technology 
Division of Argonne National Laboratory, and EPSRC Grants \#EP/G035954/1 and EP/J021172/1, and DTR Agency Grant HDTRA1-12-1-003.
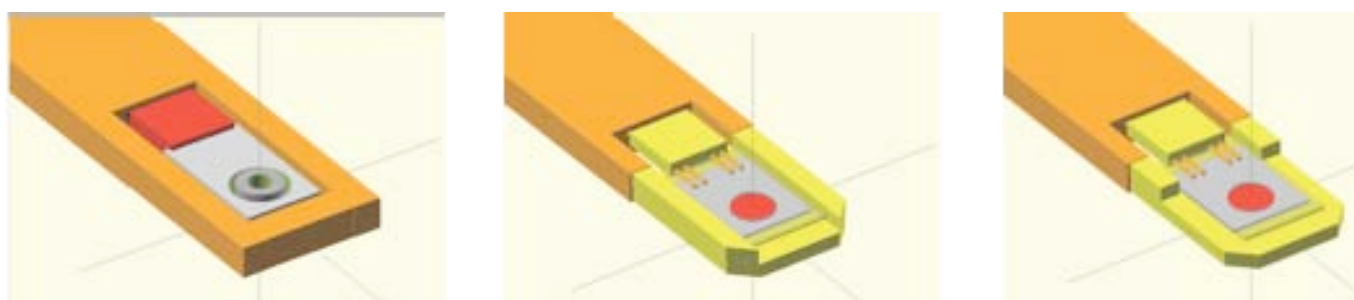

$1 \mathrm{~A}$

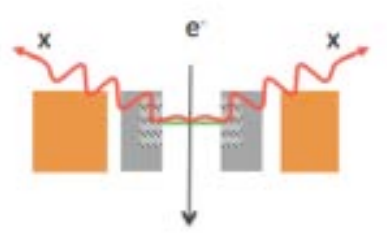

$1 \mathrm{~B}$
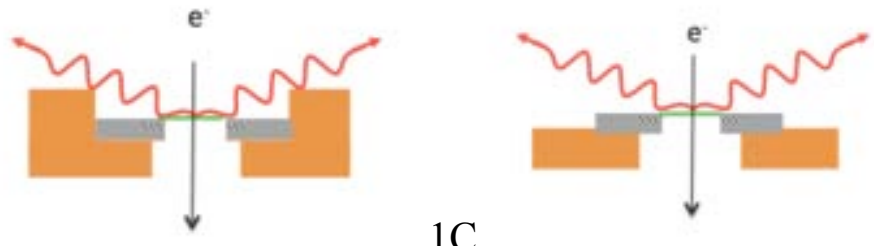

Figure 1. A, Furnace Heater Geometry; B, Membrane Heater Geometry; C, Modified Membrane Geometry.

$2 \mathrm{~A}$
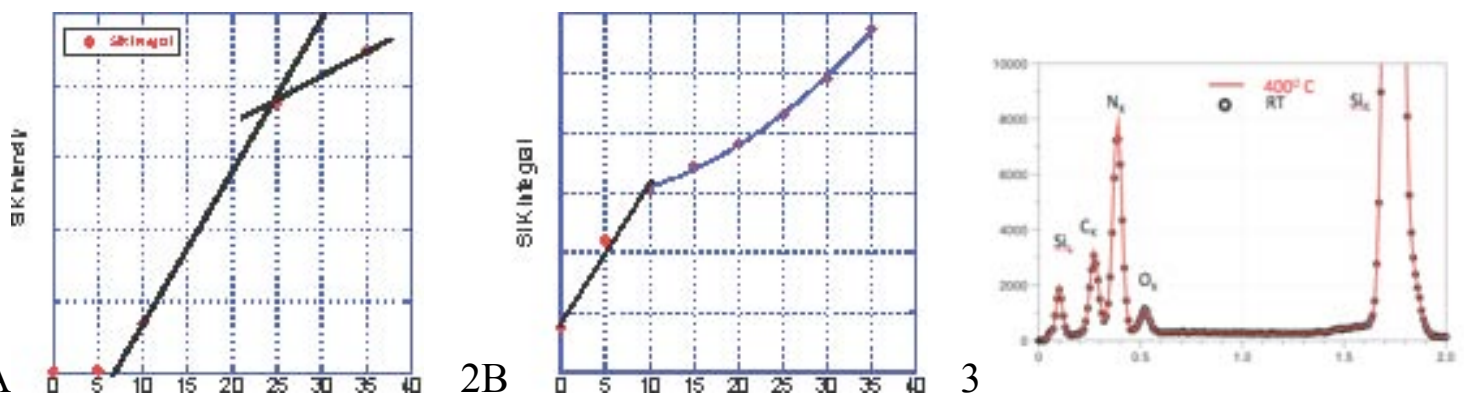

Figure 2. A, Standard Geometry; B, Modified Geometry. Figure 3. Comparison 25 vs $400 \mathrm{C}^{\mathrm{o}}$.

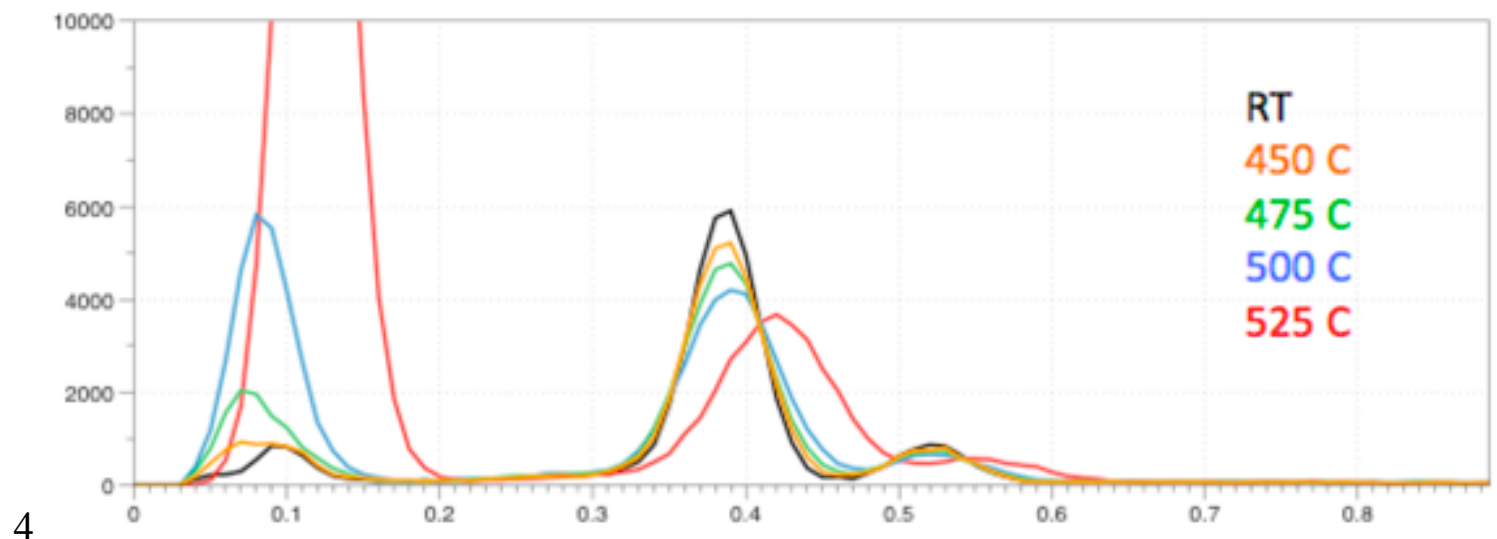

Figure 4. Effects to the low energy XEDS data due to IR signal at elevated temperatures. 